\title{
Unreduced Dynamic Complexity: Towards the Unified Science of Intelligent Communication Networks and Software
}

\author{
Andrei P. Kirilyuk \\ Solid State Theory Department, Institute of Metal Physics \\ 36 Vernadsky Avenue, $03142 \mathrm{Kiev}-142$, Ukraine \\ kiril@metfiz.freenet.kiev.ua
}

\begin{abstract}
Operation of autonomic communication network with complicated user-oriented functions should be described as unreduced many-body interaction process. The latter gives rise to complex-dynamic behaviour including fractally structured hierarchy of chaotically changing realisations. We recall the main results of the universal science of complexity based on the unreduced interaction problem solution and its application to various real systems, from nanobiosystems and quantum devices to intelligent networks and emerging consciousness. We concentrate then on applications to autonomic communication leading to fundamentally substantiated, exact science of intelligent communication and software. It aims at unification of the whole diversity of complex information system behaviour, similar to the conventional, "Newtonian" science order for sequential, regular models of system dynamics. Basic principles and first applications of the unified science of complex-dynamic communication networks and software are outlined to demonstrate its advantages and emerging practical perspectives.
\end{abstract}

\section{Introduction}

Although any information processing can be described as interaction between participating components, communication and software tools used today tend to limit this interaction to unchanged, preprogrammed system configuration, which reduces underlying interaction processes to a very special and rather trivial type of a sequential and regular "Turing machine". It does not involve any genuine novelty emergence (with the exception of thoroughly avoided failures), so that the occurring "normal" events do not change anything in system configuration and are supposed to produce only expected modification in particular register content, etc. In terms of fundamental science, one deals here with a rare limiting case of exactly solvable, integrable and certainly computable interaction problem and dynamics. The latter property, associated with total regularity, predictabil$i t y$, and decidability, forms even a major purpose and invariable operation principle of all traditional information processing and communication systems (it can be generalised to all man-made tools and engineering approaches). One can say also that usual ICT systems do not possess any genuine freedom, or unre-

Please use the following format when citing this chapter:

Kirilyuk, A. P., 2007, in IFIP International Federation for Information Processing, Volume 229, Network Control and Engineering for QoS, Security, and Mobility, IV, ed. Gaïti, D., (Boston: Springer), pp. 1-20. 
duced, decision-taking autonomy, which is totally displaced towards the human user side. One should not confuse that truly autonomic, independent kind of action with a high degree of man-made automatisation that can only (usefully) imitate a decision making process, while preserving the basic predictability and computability of such "intelligent" machine behaviour.

In the meanwhile, unreduced interaction processes involving essential and noncomputable system configuration change conquer practically important spaces at a catastrophically growing rate. On one hand, this rapid advance of unreduced interaction complexity occurs inevitably in communication networks and related software themselves as a result of strong increase of user numbers, interests, and functional demands (i.e. desired "quality of service"). On the other hand, it is due to simultaneous, and related, growth of popularity of complexdynamical applications of ICT tools, which can be truly efficient only if those tools possess corresponding levels of unreduced, interaction-driven complexity and autonomy. It can appear either as unacceptably growing frequency of complicated system failures, or else as the transition to a fundamentally different operation mode that uses inevitable uncertainties of complex interaction dynamics to strongly increase system performance quality.

The second aspect has its deep, objective origin in today's specific moment of development, where one can empirically modify, for the first time in history, the whole scale of natural system complexity at all its levels, from ultimately small, quantum systems (high-energy physics) to the most complicated, biological, ecological, and conscious systems (genetics, industrial transformation, social and psychological changes). However, the genuine understanding of complex dynamics we strongly modify is persistently missing from modern science, which gives rise to multiple and real dangers (c.f. [1]) and determines the urgency of complexity transition in major ICT instruments of progress. In fact, any progress becomes blocked when zero-complexity, regular computing instruments encounter the unreduced, noncomputable complexity of real systems.

Those reasons determine increased attention to autonomic, complex, "bioinspired" operation of communication networks and software tools that appears within various overlapping research initiatives, such as "pervasive computing", "ambient intelligence", "autonomic communication networks", "knowledgebased networks", "context awareness", "semantic grid/web", "complex software", etc. (see e.g. [2-8] for overview and further references). In fact, such kind of development has become a major, rapidly growing direction of ICT research. However, as big volumes of "promising" results and "futuristic" expectations accumulate, the need for a unified and rigorous framework, similar to exact science for non-complex, regular models, becomes evident.

Although creation of "intelligent" ICT structures has started from applied, engineering studies (contrary to traditional science applications), the emerging qualitative novelties call for a new kind of fundamental knowledge as a basis for those practically oriented efforts, without which there is a serious risk to "miss the point" in unlimited diversity of unreliable guesses. The created proto-science state of autonomic communication and complex software should 
now be extended to the genuine scientific knowledge of a new kind. This exact science should provide rigorously specified and unified description of real, not "model", complex systems with unreduced interaction of "independent" components, leading to explicitly emerging, "unexpected" structures and properties.

One can call such unreduced interaction process generalised autonomic network, even though it is realised eventually in any kind of unreduced interaction process, including complex software systems. We deal here with an important quality of new knowledge, absent in the model-based and intrinsically split usual science: once the truly consistent understanding of real complex interaction is established, it should automatically be applicable to any other, maybe externally quite different case of equally complex many-body interaction. ${ }^{1}$ Thus, truly autonomic communication networks may show basically the same patterns of behaviour and evolution as full-scale nanobiosystems, biological networks in living organisms and ecosystems, or intelligent neural networks. It is a fortunate and non-occasional circumstance, since those complex-dynamic ICT tools will be properly suited to their material basis and application objects, complex systems from the "real world", so that finally there is no separation and basic difference between complex behaviour of "natural", real-world and "artificial", ICT systems: everything tends to be irreducibly complex, interconnected, and man-made, whereupon the necessity of a unified and realistic understanding of emerging new, explicitly complex world becomes yet more evident.

Note again the essential difference with the prevailing usual science approach, where the unreduced complexity of real structures is artificially reduced, i.e. completely destroyed, so that "computer simulation" of such basic structures as elementary particles or biomolecules takes the highest existing "supercomputer" (or grid) powers and still cannot provide a really useful, unambiguous result. In view of such situation one can have justified doubts in the ability of usual, scholar science to describe truly complex systems without losing the underlying rigour (objectivity) of the scientific method itself (see e.g. [1,9-12]).

In this report we describe a theory of arbitrary many-body interaction leading to a universally applicable concept of dynamic complexity [13-26] (Sect. 2). The resulting universal science of complexity provides a working prototype of the new kind of science claimed by modern development of information and communication systems [21]. We then specify its application to emerging complex software and autonomic communication systems (Sect. 3). We finally summarise the key features of the "new mathematics of complexity" as a fundamental and rigorous basis for the new science of intelligent information systems (Sect. 4) and draw major development perspectives (Sect. 5).

${ }^{1}$ This difference from the usual, "Newtonian" science (including its versions of "new physics" and "science of complexity") stems from the fact that it does not propose any solution to the unreduced, realistic interaction problem and does not really aim at obtaining the related complete explanation of the analysed phenomena, replacing it with a mathematically "exact" (analytically "closed"), but extremely simplified, unrealistic, and often guessed "models" and "postulates", which are then mechanically adjusted to separate, subjectively chosen observation results. 


\section{Complex Dynamics of Unreduced Interaction Process}

\subsection{Multivalued Dynamics and Unified Complexity Concept}

We begin with a general equation for arbitrary system dynamics (or many-body problem), called here existence equation and simply fixing the fact of interaction between the system components (it generalises various model equations):

$$
\left\{\sum_{k=0}^{N}\left[h_{k}\left(q_{k}\right)+\sum_{l>k}^{N} V_{k l}\left(q_{k}, q_{l}\right)\right]\right\} \Psi(Q)=E \Psi(Q),
$$

where $h_{k}\left(q_{k}\right)$ is the "generalised Hamiltonian" of the $k$-th system component, $q_{k}$ is the degree(s) of freedom of the $k$-th component, $V_{k l}\left(q_{k}, q_{l}\right)$ is the (arbitrary) interaction potential between the $k$-th and $l$-th components, $\Psi(Q)$ is the system state-function, $Q \equiv\left\{q_{0}, q_{1}, \ldots, q_{N}\right\}, E$ is the generalised Hamiltonian eigenvalue, and summations are performed over all $(N)$ system components. The generalised Hamiltonian, eigenvalues, and interaction potential represent a suitable measure of dynamic complexity defined below and encompassing practically all "observable" quantities (action, energy/mass, momentum, information, entropy, etc.). Therefore (1) can express unreduced interaction configuration of arbitrary communication/software system. If interaction potential (system configuration) depends explicitly on time, one should use a time-dependent form of (1), where eigenvalue $E$ is replaced with the partial time derivative operator.

It is convenient to separate one of the degrees of freedom, e.g. $q_{0} \equiv \xi$, representing a naturally selected, usually "system-wide" entity, such as component coordinates or "connecting agent" (here now $Q \equiv\left\{q_{1}, \ldots, q_{N}\right\}$ and $k, l \geq 1$ ):

$$
\left\{h_{0}(\xi)+\sum_{k=1}^{N}\left[h_{k}\left(q_{k}\right)+V_{0 k}\left(\xi, q_{k}\right)+\sum_{l>k}^{N} V_{k l}\left(q_{k}, q_{l}\right)\right]\right\} \Psi(\xi, Q)=E \Psi(\xi, Q) \text {, }
$$

We express the problem in terms of known free-component solutions for the "functional", internal degrees of freedom of system elements $(k \geq 1)$ :

$$
\begin{gathered}
h_{k}\left(q_{k}\right) \varphi_{k n_{k}}\left(q_{k}\right)=\varepsilon_{n_{k}} \varphi_{k n_{k}}\left(q_{k}\right) \\
\Psi(\xi, Q)=\sum_{n} \psi_{n}(\xi) \varphi_{1 n_{1}}\left(q_{1}\right) \varphi_{2 n_{2}}\left(q_{2}\right) \ldots \varphi_{N n_{N}}\left(q_{N}\right) \equiv \sum_{n} \psi_{n}(\xi) \Phi_{n}(Q),
\end{gathered}
$$

where $\left\{\varepsilon_{n_{k}}\right\}$ are the eigenvalues and $\left\{\varphi_{k n_{k}}\left(q_{k}\right)\right\}$ eigenfunctions of the $k$-th component Hamiltonian $h_{k}\left(q_{k}\right)$, forming the complete set of orthonormal functions, $n \equiv\left\{n_{1}, \ldots, n_{N}\right\}$ runs through all possible eigenstate combinations, and $\Phi_{n}(Q) \equiv \varphi_{1 n_{1}}\left(q_{1}\right) \varphi_{2 n_{2}}\left(q_{2}\right) \ldots \varphi_{N n_{N}}\left(q_{N}\right)$ by definition. The system of equations for $\left\{\psi_{n}(\xi)\right\}$ is obtained then in a standard way, using the eigen-solution orthonormality (e.g. by multiplication by $\Phi_{n}^{*}(Q)$ and integration over $Q$ ):

$$
\begin{aligned}
& {\left[h_{0}(\xi)+V_{00}(\xi)\right] \psi_{0}(\xi)+\sum_{n n} V_{0 n}(\xi) \psi_{n}(\xi)=\eta \psi_{0}(\xi)} \\
& {\left[h_{0}(\xi)+V_{n n}(\xi)\right] \psi_{n}(\xi)+\sum_{n^{\prime} \neq n}^{n} V_{n n^{\prime}}(\xi) \psi_{n^{\prime}}(\xi)=\eta_{n} \psi_{n}(\xi)-V_{n 0}(\xi) \psi_{0}(\xi),}
\end{aligned}
$$


where $n, n^{\prime} \neq 0$ (also below), $\eta \equiv \eta_{0}=E-\varepsilon_{0}, \eta_{n}=E-\varepsilon_{n}, \varepsilon_{n}=\sum_{k} \varepsilon_{n_{k}}$,

$$
\begin{gathered}
V_{n n^{\prime}}(\xi)=\sum_{k}\left[V_{k 0}^{n n^{\prime}}(\xi)+\sum_{l>k} V_{k l}^{n n^{\prime}}\right], \\
V_{k 0}^{n n^{\prime}}(\xi)=\int_{\Omega_{Q}} d Q \Phi_{n}^{*}(Q) V_{k 0}\left(q_{k}, \xi\right) \Phi_{n^{\prime}}(Q), \\
V_{k l}^{n n^{\prime}}(\xi)=\int_{\Omega_{Q}} d Q \Phi_{n}^{*}(Q) V_{k l}\left(q_{k}, q_{l}\right) \Phi_{n^{\prime}}(Q),
\end{gathered}
$$

and we have separated the equation for $\psi_{0}(\xi)$ describing the generalised "ground state" of system elements, i. e. the state with minimum complexity. The obtained system of equations expresses the same problem as the starting equation (2) but now in terms of "natural" variables, and therefore it results from various starting models, including time-dependent and formally "nonlinear" ones.

We can try to solve the "nonintegrable" system of equations (5) with the help of generalised effective, or optical, potential method [27], where one expresses $\psi_{n}(\xi)$ through $\psi_{0}(\xi)$ from equations for $\psi_{n}(\xi)$ using the standard Green function technique and then inserts the result into the equation for $\psi_{0}(\xi)$, obtaining thus the effective existence equation that contains explicitly only "integrable" degrees of freedom $(\xi)[13-17,25,26]$ :

$$
h_{0}(\xi) \psi_{0}(\xi)+V_{\text {eff }}(\xi ; \eta) \psi_{0}(\xi)=\eta \psi_{0}(\xi)
$$

where the operator of effective potential $(E P), V_{\text {eff }}(\xi ; \eta)$, is given by

$$
\begin{gathered}
V_{\text {eff }}(\xi ; \eta)=V_{00}(\xi)+\hat{V}(\xi ; \eta), \quad \hat{V}(\xi ; \eta) \psi_{0}(\xi)=\int_{\Omega_{\xi}} d \xi^{\prime} V\left(\xi, \xi^{\prime} ; \eta\right) \psi_{0}\left(\xi^{\prime}\right) \\
V\left(\xi, \xi^{\prime} ; \eta\right)=\sum_{n, i} \frac{V_{0 n}(\xi) \psi_{n i}^{0}(\xi) V_{n 0}\left(\xi^{\prime}\right) \psi_{n i}^{0 *}\left(\xi^{\prime}\right)}{\eta-\eta_{n i}^{0}-\varepsilon_{n 0}}, \quad \varepsilon_{n 0} \equiv \varepsilon_{n}-\varepsilon_{0}
\end{gathered}
$$

and $\left\{\psi_{n i}^{0}(\xi)\right\},\left\{\eta_{n i}^{0}\right\}$ are complete sets of eigenfunctions and eigenvalues of a truncated system of equations:

$$
\left[h_{0}(\xi)+V_{n n}(\xi)\right] \psi_{n}(\xi)+\sum_{n^{\prime} \neq n} V_{n n^{\prime}}(\xi) \psi_{n^{\prime}}(\xi)=\eta_{n} \psi_{n}(\xi)
$$

Since the unreduced EP (10)-(11) depends essentially on the eigen-solutions to be found, the problem remains "nonintegrable" and formally equivalent to the initial formulation $(1),(2),(5)$. However, it is the effective version of a problem that reveals the nontrivial properties of its unreduced solution. The most important property of the unreduced interaction result (9)-(12) is its dynamic 
multivaluedness meaning that one has a redundant number of different but individually complete, and therefore mutually incompatible, problem solutions describing equally real system configurations. We therefore call each of them realisation of the system and problem. Plurality of system realisations follows from the dynamically nonlinear EP dependence on the solutions to be found, reflecting the evident plurality of interacting eigen-mode combinations [13-26].

It is important that dynamic multivaluedness emerges only in the unreduced problem formulation, whereas the standard theory, including usual EP method applications (see e.g. [27]) and the scholar "science of complexity" (theory of chaos, self-organisation, etc.), resorts invariably to one or another version of perturbation theory, whose "mean-field" approximation, providing an "exact", closed-form solution, totally kills dynamic redundance by eliminating the nonlinear dynamical links in (9)-(11) and retaining only one, "averaged" solution, usually expressing but small deviations from imposed system configuration:

$$
\left[h_{0}(\xi)+V_{n n}(\xi)+\tilde{V}_{n}(\xi)\right] \psi_{n}(\xi)=\eta_{n} \psi_{n}(\xi)
$$

where $\left|V_{0}(\xi)\right|<\left|\tilde{V}_{n}(\xi)\right|<\left|\sum_{n^{\prime}} V_{n n^{\prime}}(\xi)\right|$. General problem solution is then obtained as an essentially linear superposition of eigen-solutions of (13) similar to (4). This dynamically single-valued, or unitary, problem reduction forms the basis of the whole canonical science paradigm.

The unreduced, truly complete general solution to a problem emerges as a dynamically probabilistic sum of redundant system realisations, each of them being roughly equivalent to the whole "general solution" of usual theory:

$$
\rho(\xi, Q)=\sum_{r=1}^{N_{\Re}} \oplus \rho_{r}(\xi, Q),
$$

where the observed (generalised) density, $\rho(\xi, Q)$, is obtained as the statefunction squared modulus, $\rho(\xi, Q)=|\Psi(\xi, Q)|^{2}$ (for "wave-like" complexity levels), or as the state-function itself, $\rho(\xi, Q)=\Psi(\xi, Q)$ (for "particle-like" structures), index $r$ enumerates system realisations, $N_{\Re}$ is realisation number (its maximum value is equal to the number of system components, $N_{\Re}=N$ ), and the sign $\oplus$ designates the special, dynamically probabilistic meaning of the sum. The latter implies that incompatible system realisations are forced, by the same driving interaction, to permanently replace each other in a causally (dynamically) random order thus consistently defined. The $r$-th realisation statefunction, $\Psi_{r}(\xi, Q)$, in the unreduced general solution (14) is obtained as

$$
\begin{gathered}
\Psi_{r}(\xi, Q)=\sum_{i} c_{i}^{r}\left[\Phi_{0}(Q) \psi_{0 i}^{r}(\xi)+\right. \\
\left.+\sum_{n, i^{\prime}} \frac{\Phi_{n}(Q) \psi_{n i^{\prime}}^{0}(\xi) \int_{\Omega_{\xi}} d \xi^{\prime} \psi_{n i^{\prime}}^{0 *}\left(\xi^{\prime}\right) V_{n 0}\left(\xi^{\prime}\right) \psi_{0 i}^{r}\left(\xi^{\prime}\right)}{\eta_{i}^{r}-\eta_{n i^{\prime}}^{0}-\varepsilon_{n 0}}\right]
\end{gathered}
$$


where $\left\{\psi_{0 i}^{r}(\xi), \eta_{i}^{r}\right\}$ are $r$-th realisation eigen-solutions of the unreduced EP equation (9) and the coefficients $c_{i}^{r}$ should be found from the state-function matching conditions at the boundary where interaction effectively vanishes. The corresponding $r$-th realisation EP takes the form (derived from (10)-(11)):

$$
\begin{gathered}
V_{\mathrm{eff}}\left(\xi ; \eta_{i}^{r}\right) \psi_{0 i}^{r}(\xi)=V_{00}(\xi) \psi_{0 i}^{r}(\xi)+ \\
+\sum_{n, i^{\prime}} \frac{V_{0 n}(\xi) \psi_{n i^{\prime}}^{0}(\xi) \int_{\Omega_{\xi}} d \xi^{\prime} \psi_{n i^{\prime}}^{0 *}\left(\xi^{\prime}\right) V_{n 0}\left(\xi^{\prime}\right) \psi_{0 i}^{r}\left(\xi^{\prime}\right)}{\eta_{i}^{r}-\eta_{n i^{\prime}}^{0}-\varepsilon_{n 0}}
\end{gathered}
$$

Equations (14)-(16) reveal, in particular, dynamic localisation of a system in any its normal, "regular" realisation around its characteristic eigenvalue and configuration (due to the resonance denominator) and reverse delocalisation during transition between regular realisations, occurring though a special, intermediate realisation of the wavefunction $[13,17,20,23,28]$ (see also below).

Direct comparison between the unreduced (9),(12),(14)-(16) and reduced (13) problem solutions reveals the exact dynamic origin and huge scale of difference between the real system complexity and its model simplification in the unitary theory. In particular, the unreduced solution (14) implies that any measured value is intrinsically unstable and will unpredictably change to another one, corresponding to another, randomly chosen realisation. Such kind of behaviour is readily observed in nature and actually explains the living organism behaviour $[13,16-18]$, but is thoroughly avoided in the unitary approach and technological systems (including ICT systems), where it is correctly associated with linear "noncomputability" and technical failure (we shall consider below that limiting regime of complex dynamics). Therefore the universal dynamic multivaluedness revealed by rigorous problem solution forms the fundamental basis for the transition to "bio-inspired" and "intelligent" kind of operation in artificial, technological and communication systems, where causal randomness can be transformed from an obstacle to a qualitative advantage (Sect. 3).

The rigorously derived randomness of the generalised EP formalism (14)(16) is accompanied by the dynamic definition of probability. As elementary realisations are equivalent in their "right to appear", the dynamically obtained, a priori probability, $\alpha_{r}$, of elementary realisation emergence is given by

$$
\alpha_{r}=\frac{1}{N_{\Re}}, \quad \sum_{r} \alpha_{r}=1 \text {. }
$$

However, a real observation may resolve only uneven groups of elementary realisations. The dynamic probability of such general, compound realisation is determined by the number, $N_{r}$, of elementary realisations it contains:

$$
\alpha_{r}\left(N_{r}\right)=\frac{N_{r}}{N_{\Re}} \quad\left(N_{r}=1, \ldots, N_{\Re} ; \sum_{r} N_{r}=N_{\Re}\right), \quad \sum_{r} \alpha_{r}=1 .
$$

An expression for expectation value, $\rho_{\exp }(\xi, Q)$, follows from $(14),(17)-(18)$ for statistically long observation periods: 
A.P. Kirilyuk

$$
\rho_{\exp }(\xi, Q)=\sum_{r} \alpha_{r} \rho_{r}(\xi, Q)
$$

It is important, however, that our dynamically derived randomness and probability need not rely on such "statistical", empirically based result, so that the basic expressions (14)-(18) remain valid even for a single event of realisation emergence and before any event happens at all.

Realisation probability distribution can be obtained in another way, involving generalised wavefunction and Born's probability rule $[13,15,17,20,23,28]$. The wavefunction describes system state during its transition between "regular", localised realisations and constitutes a particular, "intermediate" realisation with extended and "loose" (chaotically changing) structure, where system components transiently disentangle before forming the next "regular" realisation. The intermediate, or "main", realisation is explicitly obtained in the unreduced EP formalism as the single, exceptional one for which the nonintegrable terms of the general EP (11),(16) become indeed small and it is reduced to a separable version of perturbative, "mean-field" type (13) $[13,15,17,20,23,28]$. This special realisation provides, in particular, the causal, realistic version of the quantum-mechanical wavefunction at the lowest, quantum levels of complexity. The "Born probability rule", now causally derived and extended to any level of world dynamics, states that realisation probability $\alpha_{r}$ is determined by wavefunction value (its squared modulus for "wave-like" complexity levels) for the respective system configuration $X_{r}: \alpha_{r}=\left|\Psi\left(X_{r}\right)\right|^{2}$. The generalised wavefunction (or distribution function) $\Psi(x)$ satisfies the universal Schrödinger equation (Sect. 2.2), rigorously derived by causal quantisation of complex dynamics, while Born's probability rule follows from the above dynamic "matching conditions" for the state-function (15), which are satisfied during transitions from regular realisations to the wavefunction and back $[13,15,17,20,23,28]$. It is only this "averaged", weak-interaction state of the wavefunction, or "main" realisation, that remains in the single-valued model and paradigm of unitary science, which explains both its partial success and basic limitations.

Closely related to dynamic redundance is dynamic entanglement of interacting components, described in (15) by the weighted products of state-function elements depending on various degrees of freedom $(\xi, Q)$. It is a rigorous expression of the tangible quality of the emerging system structure, absent in unitary models. The obtained dynamically multivalued entanglement describes a "living" structure, permanently changing and probabilistically adapting its configuration, which endows "bio-inspired" and "autonomic" technologies with a wellspecified basis. The properties of dynamically multivalued entanglement and adaptability are amplified due to probabilistic fractality of the unreduced problem solution $[13,16-18,20]$, essentially extending usual, single-valued fractality and obtained by application of the same EP method to solution of the truncated system of equations (12) used in the first-level EP expression (11),(16).

We can now consistently and universally define the unreduced dynamic complexity, $C$, of any real system (or interaction process) as arbitrary growing function of the total number, $N_{\Re}$, of explicitly obtained system realisations or the 
rate of their change, $C=C\left(N_{\Re}\right), \quad d C / d N_{\Re}>0$, equal to zero for the unrealistic case of only one system realisation, $C(1)=0$. Suitable examples are provided by $C\left(N_{\Re}\right)=C_{0} \ln N_{\Re}$, generalised energy/mass (temporal rate of realisation change), and momentum (spatial rate of realisation emergence) [13-25]. It becomes clear now that the whole dynamically single-valued paradigm and results of the canonical theory (including its versions of "complexity" and e.g. "multi-stability") correspond to exactly zero value of the unreduced dynamic complexity, which is equivalent to the effectively zero-dimensional, point-like projection of reality from the "exact-solution" perspective (cf. [9-12]).

Correspondingly, any dynamically single-valued "model" is strictly regular and cannot possess any true, intrinsic randomness (chaoticity), which can only be introduced artificially, e.g. as a regular "amplification" of a "random" (by convention) external "noise". By contrast, our unreduced dynamic complexity is practically synonymous to the equally universal and intrinsic chaoticity, since multiple system realisations appearing and disappearing in the real space (and thus forming its tangible, changing structure) are redundant (mutually incompatible), which is the origin of both complexity and chaoticity. The genuine dynamical chaos thus obtained has a complicated internal structure (contrary to ill-defined unitary "stochasticity") and always contains partial regularity, which is dynamically, inseparably entangled with truly random elements.

The universal dynamic complexity, chaoticity, and related properties involve the essential, or dynamic, nonlinearity of the unreduced problem solution and system behaviour. It is provided by dynamical links of the developing interaction process, as they are expressed in EP dependence on the eigen-solutions to be found (see (9)-(11),(16)). It is the dynamically emerging and irreducible nonlinearity, since it appears inevitably even for a "linear" initial problem expression (1)-(2),(5), whereas usual, mechanistic "nonlinearity" is but an imposed, dispensable imitation of the essential EP nonlinearity. Essential nonlinearity leads to the omnipresent dynamic instability of any system state (realisation), since both are determined by the same dynamic feedback mechanism.

Universality of our description leads, in particular, to the unified understanding of the whole diversity of dynamical regimes and structures [13,14,17,19-22]. One standard, limiting case of complex (multivalued) dynamics, called uniform, or global, chaos, is characterised by essentially different realisations with a homogeneous probability distribution $\left(N_{r} \approx 1, \alpha_{r} \approx 1 / N_{\Re}\right.$ for all $r$ in (18)) and occurs when major parameters of interacting entities (suitably represented by frequencies) have close values (which leads to a strong "conflict of interests" and resulting "deep disorder"). The complementary limiting regime of multivalued self-organisation, or self-organised criticality (SOC) emerges for sufficiently different parameters of interaction components, so that a small number of relatively rigid, low-frequency components "enslave" a hierarchy of high-frequency and rapidly changing, but configurationally similar, realisations (i.e. $N_{r} \sim N_{\Re}$ and realisation probability distribution is highly uneven). The difference of this extended, multivalued self-organisation (and SOC) from usual, unitary version is essential: despite the rigid external shape of system configuration in this 
regime, it contains the intense "internal life" and chaos of permanently changing "enslaved" realisations (which are not superposable unitary "modes"). In this sense the generalised SOC structure, and with it the whole unreduced complexity, can be described as confined chaos, where global chaos has the lowest and quasi-regular SOC the highest degree of chaos confinement.

Another advance with respect to unitary "science of complexity" is that the unreduced, multivalued self-organisation unifies the essentially extended versions of a whole series of separated unitary "models", including "selforganisation", "synergetics", SOC, any "synchronisation", "control of chaos", "attractors", and "mode locking". All intermediate dynamic regimes between those two limiting cases of uniform chaos and quasi-regular SOC, as well as their multi-level combinations, are obtained for respective parameter values.

The point of transition to the strong chaos is expressed by the universal criterion of global chaos onset:

$$
\kappa \equiv \frac{\Delta \eta_{i}}{\Delta \eta_{n}}=\frac{\omega_{\xi}}{\omega_{q}} \cong 1
$$

where $\kappa$ is the introduced chaoticity parameter, $\Delta \eta_{i}, \omega_{\xi}$ and $\Delta \eta_{n} \sim \Delta \varepsilon, \omega_{q}$ are energy-level separations and frequencies for inter-component and intracomponent motions, respectively. At $\kappa \ll 1$ one has an externally regular multivalued SOC regime, which degenerates into global chaos as $\kappa$ grows from 0 to 1 , and maximum irregularity at $\kappa \approx 1$ is again transformed into a SOC kind of structure (but with a "reversed" configuration) at $\kappa \gg 1$.

One can compare this transparent and universal picture with the existing diversity of separated and incomplete unitary criteria of chaos and regularity. Only the former provide a real possibility of understanding and control of ICT systems of arbitrary complexity, where more regular, SOC regimes can serve for (loose) control of system dynamics, while less regular ones can also play a positive role of efficient search and adaptation means. This combination forms the basis of any "biological" and "intelligent" kind of behaviour [13,16-18, 20-22] and therefore can constitute the essence of intelligent ICT paradigm supposed to extend the now realised (quasi-) regular kind of operation in the uttermost limit of SOC $(\kappa \rightarrow 0)$. While the latter inevitably becomes inefficient with growing system sophistication (where the chaos-bringing resonances of (20) cannot be avoided), it definitely lacks the "intelligent power" of unreduced complex dynamics to generate meaning and adaptable structure development.

\subsection{Huge Efficiency of Unreduced Complex Dynamics and Universal Symmetry of Complexity}

Dynamically probabilistic fractality is the intrinsic property of unreduced interaction development $[13,16-18,20]$. It is obtained by application of the same EP method (9)-(11) to the truncated system of equations (12), then to the next truncated system, etc., which gives the irregular and probabilistically adapting hierarchy of realisations showing the intermittent mixture of global chaos 
and regularity, or confined randomness (Sect. 2.1). The total realisation number $N_{\Re}$, and thus operation power, of this autonomously branching interaction process with a dynamically parallel structure grows exponentially within any time period. It can be estimated in the following way [17-21].

If our system of inter-connected elements contains $N_{\text {unit }}$ "processing units", or "junctions", and if each of them has $n_{\text {conn }}$ real or "virtual" (possible) links, then the total number of interaction links is $N=n_{\text {conn }} N_{\text {unit }}$. In most important cases $N$ is a huge number: for both human brain and genome interactions $N$ is greater than $10^{12}$, and being much more variable for communication/software systems, it can easily grow to similar "astronomical" ranges. The key property of unreduced, complex interaction dynamics, distinguishing it from any unitary version, is that the maximum number $N_{\Re}$ of realisations taken by the system (also per time unit) and determining its real "power" $P_{\text {real }}$ (of search, memory, cognition, etc.) is given by the number of all possible combinations of links, i.e.

$$
P_{\text {real }} \propto N_{\Re}=N ! \rightarrow \sqrt{2 \pi N}\left(\frac{N}{e}\right)^{N} \sim N^{N} \gg \gg N .
$$

Any unitary, sequential model of the same system (including its mechanistically "parallel" and "complex" modes) would give $P_{\text {reg }} \sim N^{\beta}$, with $\beta \sim 1$, so that

$$
P_{\text {real }} \sim\left(P_{\text {reg }}\right)^{N} \gg \gg P_{\text {reg }} \sim N^{\beta} \text {. }
$$

Thus, for $N \sim 10^{12}$ we have $P_{\text {real }} \gg 10^{10^{13}} \gg 10^{10^{12}} \sim 10^{N} \rightarrow \infty$, which is a "practical infinity", also with respect to the unitary power of $N^{\beta} \sim 10^{12}$.

These estimates demonstrate the true power of complex (multivalued) communication dynamics that remains suppressed within the now dominating unitary, quasi-regular operation mode. Huge power of complex-dynamical interaction correlate with the new quality emergence, such as intelligence and consciousness (at higher levels of complexity) $[17,20]$, in direct relation to our intelligent communication paradigm meaning that such properties as sensi$b l e$, context-related information processing, personalised understanding and autonomous creativity (useful self-development), desired for the new ICT systems, are inevitable qualitative manifestations of the above "infinite" power.

Everything comes at a price, however, and a price to pay for the above qualitative advantages is rigorously specified as irreducible dynamic randomness and thus unpredictability of operation details of complex information-processing systems. We rigorously confirm here an evident idea that autonomous adaptability and genuine creativity exclude any detailed, regular programming in principle. But then what can serve as a guiding principle and practical construction strategy for those qualitatively new communications networks and their intelligent elements? We show that guiding rules and strategy are determined by a general law of real dynamics, the universal symmetry, or conservation, of complexity [13,15-18,20-24]. This universal "order of nature" unifies the extended versions of all usual (correct) laws, symmetries, and principles (now causally derived and realistically interpreted). Contrary to any unitary symmetry, the 
universal symmetry of complexity is irregular in its structure, but always exact (never "broken"). Its "horizontal" manifestation (at a given complexity level) implies dynamic transformation of the system between its changing realisations, as opposed to abstract "symmetry operator" idea. Therefore the symmetry of system complexity totally determines its dynamics and expresses the deep connection between often visibly dissimilar and chaotically changing configurations.

Another, "vertical" manifestation of the symmetry of complexity determines emergence and development of different complexity levels of a real interaction. System "potentiality", or real power to create new structure is universally described by a form of complexity called dynamic information and generalising usual "potential energy" $[13,15,17,20,24]$. This potential, latent complexity is transformed, during interaction development, into explicit, "unfolded" form of dynamic entropy (generalising kinetic, or heat, energy). Universal conservation of complexity means that this important transformation, determining every system dynamics and evolution, preserves the sum of dynamic information and entropy, or total complexity (for a given system or process). This universal formulation of the symmetry of complexity includes its above "horizontal" manifestation and, for example, extended and unified versions of the first and second laws of thermodynamics (i.e. conservation of energy by its permanent degradation). It also helps to eliminate persisting series of confusions around information, entropy, and complexity in the unitary theory (thus, any real, useful "information" is expressed rather by our dynamic entropy $[13,17]$ ).

It is not difficult to show $[13,15,17,20,23,24]$ that a universal measure of dynamic information is provided by action $\mathcal{A}$ known from classical mechanics, but now acquiring a universal, essentially nonlinear and causally complete meaning. One obtains then the universal expression of complexity conservation law in the form of generalised Hamilton-Jacobi equation for $\mathcal{A}=\mathcal{A}(x, t)$ :

$$
\left.\frac{\Delta \mathcal{A}}{\Delta t}\right|_{x=\text { const }}+H\left(x,\left.\frac{\Delta \mathcal{A}}{\Delta x}\right|_{t=\text { const }}, t\right)=0,
$$

where the Hamiltonian, $H=H(x, p, t)$, considered as a function of emerging space coordinate $x$, momentum $p=\left.(\Delta \mathcal{A} / \Delta x)\right|_{t=\text { const }}$, and time $t$, expresses the unfolded, entropy-like form of differential complexity, $H=\left.(\Delta S / \Delta t)\right|_{x=\text { const }}$ (note that discrete, rather than continuous, versions of derivatives here reflect the quantised character of unreduced complex dynamics $[13,15,17,20,23,24])$. As in the naturally dualistic multivalued dynamics every localised, "regular" realisation is transformed into the extended wavefunction and back (Sect. 2.1), one obtains also the universal Schrödinger equation for the generalised wavefunction (or distribution function) $\Psi(x, t)$ by applying causal quantisation procedure $[13,15,17,20,23,24]$ to the Hamilton-Jacobi equation (23):

$$
\mathcal{A}_{0} \frac{\partial \Psi}{\partial t}=\hat{H}\left(x, \frac{\partial}{\partial x}, t\right) \Psi
$$

where $\mathcal{A}_{0}$ is a characteristic action value (equal to Planck's constant at the lowest, quantum levels of complexity) and the Hamiltonian operator, $\hat{H}$, is ob- 
tained from the Hamiltonian function $H=H(x, p, t)$ of equation (23) with the help of causal quantisation (we put continuous derivatives here for simplicity).

Equations (23)-(24) represent the universal differential expression of the symmetry of complexity showing how it determines dynamics and evolution of any system or interaction process (they also justify our use of the Hamiltonian form for the starting existence equation (1)-(2)). This universally applicable Hamilton-Schrödinger formalism can be useful for rigorous description of any complex interaction network, provided we find its truly complete (dynamically multivalued) solution with the help of unreduced EP method (Sect. 2.1).

\section{Unified Science of Complex ICT Systems}

\subsection{Main Principles of Complex ICT System Operation and Design}

The rigorously derived framework of the Universal Science of Complexity (Sect. 2) finds its further confirmation in numerous applications at different complexity levels, from fundamental physics and cosmology (Quantum Field Mechanics) $[13,17,23-25,28]$ to living organism dynamics (causally specified genomics and nanobiotechnology) [13,16-19], ecological system development (realistic sustainability concept) $[13,22]$, and theory of emergent true intelligence and consciousness $[13,17,20]$ (see also Sect. 4). These results give a realistic hope for an equally successful application of the same complexity concept to the new generation of communication and software systems with "bio-inspired" and "intelligent" properties [2-7], which are actually indispensable for efficient work with the critically emerging real-system complexity from the above applications (Sect. 1). We provide here an outline of the main principles of expected behaviour and design of complex-dynamic communication networks and software as they follow from the universal complexity framework (Sect. 2).

The single unifying principle of complex system dynamics and evolution is provided by the universal symmetry of complexity describing complexity conservation by its permanent transformation from dynamic information into entropy as the unified structure and purpose of any system evolution (Sect. 2.2). While the very existence of such unified law is important for efficient analysis of generalised autonomic networks (Sect. 1), we can specify now the ensuing particular principles that can be especially useful for their practical design and control.

We start with the complexity correspondence principle that directly follows from the universal symmetry of complexity and takes various forms for different application tasks $[13,17,20]$. A general enough formulation maintains that any interaction between complex systems (e.g. within a "global" system) tend to have maximum efficiency for comparable values of interacting system complexities (see Sect. 2.1 for the universal complexity definition). Moreover, interaction components with higher complexity tend to "enslave", or control, those with lower complexity within a resulting SOC-type state (Sect. 2.1), while very close complexities of interacting components often give rise to global chaoticity. 
It follows that in order to increase efficiency one should use tools of certain complexity for control of structures of comparable and slightly lower complexity. Lower-complexity tools cannot correctly control or even simulate highercomplexity behaviour in principle, while a much higher complexity tool will produce a lot of unnecessary activity during control of low-complexity structure. This "simple" rule has a rigorous, reliable basis and helps to avoid any inefficient solution (e.g. usual unconditional, "total" elimination of randomness).

Reproduction, or simulation, of a system behaviour by another, "computing" or controlling system can also be successful only if the simulating system has superior complexity, which immediately shows, for example, that any quantum device could at best simulate or compute only another quantum, but not classical, localised and deterministic behaviour, which provides a rigorous proof of impossibility of real quantum computation $[13,17]$. Losses produced by the dominating neglect of underlying hierarchy of complexity are evident and include a similar situation in nanotechnology (cf. [19]). Another use of complexity correspondence involves popular ideas of "context-based" information technologies, where the necessity of unreduced dynamic complexity in ICT systems becomes evident, as any human "context" has a high enough complexity.

The above rule of control (or "enslavement") of lower-complexity dynamics by a higher-complexity tool can be extended to a general principle of complexdynamical control. Contrary to unitary control schemes (e.g. "control of chaos"), the realistically substantiated complex-dynamical control paradigm shows that any resulting, "controlled" dynamics cannot be regular, i.e. "totally controlled", as it is implied by the unitary control idea leading to unpredictably (and inevitably) emerging catastrophic failures of any technological systems with usual, "protective" control design. In reality one always obtains a dynamically multivalued, internally chaotic SOC state, and the general purpose of complexdynamic, reality-based control is to ensure optimal, quasi-free development of the global system complexity, including gentle, "orienting" actions of control that cannot be separated from the controlled system dynamics and should be considered within a unified, unreduced interaction analysis (Sect. 2). In that way one can realistically obtain a failure-proof, catastrophe-free systems that will avoid big crashes by using creative power of small, interaction-driven irregularities, quite similar to unreduced life dynamics, now causally understood [13,16-18].

This brings us to the principle of huge creative power of unreduced complex dynamics as it is described above (Sect. 2.2). It implies, practically, that designing truly autonomous and intelligent ICT systems, one should "liberate" them to go freely, by their own way, unpredictable in its chaotically varying details, to the well-defined general purpose of maximum complexity-entropy obtained at the expense of inserted dynamical information that replaces usual deterministic programme. One obtains thus the exponentially huge, practically infinite gain in efficiency with respect to unitary, sequential operation due to interactive adaptability of probabilistic fractal of dynamically emerging links [17-21] (Sect. 2.2). In exchange, one should accept the omnipresent, massive, unavoidable uncertainty of unreduced interaction dynamics. It can, however, be properly confined 
and constructively used by alternating uniformly chaotic (irregular, "searching") and SOC (regular, "fixing") states, whose separation is governed by our unified chaoticity criterion (20) in terms of major system resonances.

In conclusion of this outline of major principles of complex autonomic networks, it would be useful to return to the global framework of the symmetry of complexity that shows, in the above way, what can happen in the strongly interactive ICT system of arbitrary complexity and how one can efficiently design and control the unreduced interaction results by its causally complete understanding. The latter can certainly be properly specified and adapted, where necessary, to any particular case of reduced, mechanistic "complexity", but now with the underlying clear understanding of the performed actions (Sect. 5).

\subsection{Complexity Transition in ICT Systems: Towards the New Era of Intelligent Communication Technology}

The qualitatively big transition from unrealistic unitary models to the unreduced, multivalued dynamics of real, massively interactive ICT systems can be designated as complexity transition. It has a narrow meaning of transition from the uttermost limit of pseudo-regular SOC of usual ICT to the fractal dynamic hierarchy of various chaotic states, according to the chaoticity criterion (20). In a wider sense, one deals with a fundamentally based change of concept of modern technology (Sects. 1, 3.1,5) and related way of development.

Unreduced complexity appearance should rather be tested first at the level of software. Using the results of complexity correspondence principle described in the previous section, we can suppose that complexity transition can be conveniently started within context-based technology, where complexity-bringing interaction involves essential, structure-changing, autonomic exchange between context-bearing elements. Their unreduced interaction should then be designed according to the principles of complex interaction dynamics (Sects. 2, 3.1).

As the unreduced interaction complexity forms a growing hierarchy of levels $[13,17,20]$, one obtains eventually a whole series of system transitions to ever growing complexity. Software version of initial complexity emergence will later involve hardware elements into structure-changing interaction processes. Communication network or its respective parts operate in that case as a single, holistic process of "generalised quantum beat" (chaotic realisation change). Transition to a high enough complexity level will bring about first elements of genuine network intelligence and then consciousness, as both these properties can be consistently explained as high enough levels of unreduced interaction complexity $[13,17,20]$. One can designate then intelligence and consciousness transitions as sufficiently high-level complexity transitions involving unreduced soft- and hardware interaction. Whereas lower-level complexity transitions can be limited to separate network parts and operation layers, such higher-level features as intelligence and (machine) consciousness will progressively involve the whole network dynamics, which is the evident highest level of communication network autonomy. Whereas already the lowest complexity transition involves 
context-bearing elements of human complexity, intelligence transition marks the beginning of inseparable entanglement of machine and human complexity development that can be the unique real way of progressive development of "natural" intelligence and consciousness $[20,21]$.

\section{New Mathematics of Complexity and Its Applications}

After having outlined, in the previous section, practically oriented principles of ICT applications of the unreduced complexity, let us now summarise purely mathematical, rigorously expressed novelties of the unreduced interaction analysis to be used in the new kind of knowledge (see also the end of paper [18]).

The new mathematics of complexity is represented by the unified, single structure of dynamically probabilistic fractal obtained as the unreduced solution of real interaction problem (Sect. 2.1). All its properties, describing the exact world structure and dynamics, are unified within the single, never broken symmetry, or conservation, of complexity including its unceasing transformation from complexity-information to complexity-entropy (Sect. 2.2).

One can emphasize several features of this unified structure and law of the new mathematics, distinguishing it essentially from unitary mathematics:

(i) Nonuniqueness of any real (interaction) problem solution taking the form of its dynamic multivaluedness (redundance). Exclusively complex-dynamic (multivalued, internally chaotic) existence of any real system (cf. conventional "existence and uniqueness" theorems).

(ii) Omnipresent, explicit emergence of qualitatively new structure and dynamic origin of time (change) and events: $a \neq a$ for any structure/element $a$ in the new mathematics and reality, while $a=a$ (self-identity postulate) in the whole usual mathematics, which thus excludes any real change in principle.

(iii) Fractally structured dynamic entanglement of unreduced problem solution (interaction-driven structure weaving within any single realisation): rigorous expression of material quality in mathematics (as opposed to "immaterial", qualitatively "neutral", "dead" structures of usual mathematics).

(iv) Basic irrelevance of perturbation theory and "exact solution" paradigm: the unreduced problem solution is dynamically random (permanently, chaotically changing), dynamically entangled (internally textured and "living") and fractal (hierarchically structured). Unified dynamic origin and causally specified meaning of nonintegrability, nonseparability, noncomputability, randomness, uncertainty (indeterminacy), undecidability, "broken symmetry", etc. Real interaction problem is nonintegrable and nonseparable but solvable. Realistic mathematics of complexity is well defined (certain, unified and complete), but its structures are intrinsically "fuzzy" (dynamically indeterminate) and properly diverse (not reduced to numbers or geometry).

(v) Dynamic discreteness (causal quantisation) of the unreduced interaction products (realisations): qualitative inhomogeneity, or nonunitarity, of any 
system structure and evolution, dynamic origin of (fractally structured) space. Qualitative irrelevance of usual unitarity, continuity and discontinuity, calculus, and all major structures (evolution operators, symmetry operators, any unitary operators, Lyapunov exponents, path integrals, etc.).

Let us recall now how these fundamental novelties of the universal science of complexity help to solve consistently real-world problems $[13-26,28]$ that accumulate and remain unsolvable within the unitary science paradigm:

(1) In particle and quantum physics one obtains causal, unified origin and structure of elementary particles, all their properties ("intrinsic", quantum, relativistic), and fundamental interactions [13,17,23,24,28]. Complex-dynamic origin of mass avoids any additional, abstract entities (Higgs bosons, zeropoint field, extra dimensions, etc.). Renormalised Planckian units provide consistent mass spectrum and other problem solution. Complex-dynamic cosmology resolves the dark mass and energy problems without "invisible" entities, together with other old and new problems of unitary cosmology.

(2) At a higher complexity sublevel of interacting particles $[13,17,23,25,26]$ one obtains genuine, purely dynamic quantum chaos for Hamiltonian (nondissipative) dynamics and correct correspondence principle. A slightly dissipative interaction dynamics leads to the causally complete understanding of quantum measurement in terms of quantum dynamics. Intrinsic classically emerges as a higher complexity level in a closed, bound system, like atom.

(3) Realistic, causally complete foundation of nanobiotechnology is provided by rigorous description of arbitrary nanoscale interaction, revealing the irreducible role of chaoticity $[17,19]$. Exponentially huge power of unreduced, complex nanobiosystem dynamics explains the essential properties of life and has direct relation to complex ICT system development (Sect. 2.2).

(4) Causally complete description of unreduced genomic interactions leads to reliable, rigorously substantiated genetics and consistent understanding of related evolutionary processes $[16,18]$.

(5) Higher-complexity applications include general many-body problem solution and related description of "difficult" cases in solid-state physics, unreduced dynamics and evolution of living organisms, integral medicine, emergent (genuine) intelligence and consciousness, complex ICT system dynamics, creative ecology and practically efficient sustainable development concept, rigorously specified ethics and aesthetics [13,16-18,20-22].

Note that only the unreduced, universal concept of complexity can be useful for real problem solution culminating in creation of complex, autonomic and intelligent ICT systems, which in their turn form a consistent, necessary basis for further control and development of real interaction complexity. 


\section{Conclusion and Perspectives}

We have presented a rigorously specified, working prototype of the unified science of complex ICT systems demonstrating its necessity, feasibility and practical application efficiency. One obtains thus a new, intrinsically unified and realistic kind of knowledge with extended possibilities of consistent understanding and progressive development of real-world complexity.

Application of the universal science of complexity to autonomic communication and information systems has a special importance among other applications, since it is the first case of totally artificial, man-made systems that can possess unreduced complexity features comparable to those of natural systems and remaining irrational "mysteries" within the unitary science framework (starting from "quantum mysteries" $[13,17,23-26,28]$ ). Successful realisation of unreduced ICT complexity will open the way to a much larger, unlimited and now reliable complexity design becoming so necessary today because of the rapid empirical technology progress (Sect. 1). Those major purposes cannot be attained within unitary imitations of communication and software complexity (e.g. $[4,8]$ ), since they avoid the unreduced, network-wide interaction analysis and use essentially simplified models even for separate component description (in particular, they cannot describe the emerging genuine chaoticity $[13,17]$ ).

It is difficult to have serious doubts about basic consistency of a unified complexity framework based on the unreduced problem solution and confirmed by a variety of applications (Sects. 2,4). Understanding of unreduced interaction complexity is indispensable for efficient design of even regular, but mechanically "complicated" systems. It is evident that all increasingly popular "bio-inspired", autonomic and "intelligent" imitations of natural complexity will be much more successful with the help of consistent understanding of the unreduced versions and properties of life, intelligence, etc. In this sense one can say that application of the universal science of complexity will certainly provide consistent clarification of what is possible or impossible in artificial complexity design, whereupon further development of ICT applications of unreduced complexity analysis can produce only positive (and urgently needed) result.

\section{References}

1. M. Rees, Our Final Hour: A Scientist's Warning: How Terror, Error, and Environmental Disaster Threaten Humankind's Future in This Century - On Earth and Beyond (Basic Books, New York, 2003).

2. The First International Workshop on Autonomic Communication (WAC 2004), http://www.autonomic-communication.org/wac/wac2004/program.html.

Proceedings are being published as LNCS No. 3457, Springer Verlag (2005).

3. European Commission FP6, FET Situated and Autonomic Communications, http://www.cordis.lu/ist/fet/comms.htm. Links to other related FET initiatives can be found at http://www.cordis.lu/ist/fet/areas.htm. 
4. S. Bullock and D. Cliff, Complexity and Emergent Behaviour in ICT Systems, Foresight Intelligent Infrastructure Systems Project, UK Office of Science and Technology (2004), http://www.foresight.gov.uk/

Intelligent_Infrastructure_Systems/Complexity_and_Emergent_Behaviour.html.

5. G. Di Marzo Serugendo, A. Karageorgos, O.F. Rana, and F. Zambonelli (Eds.), Engineering Self-Organising Systems: Nature Inspired Approaches to Software Engineering, LNCS No. 2977 (Springer Verlag, Berlin, 2004).

6. T. Berners-Lee, K. Hendler, and O. Lassila, The Semantic Web, Scientific American, May (2001) 35-43.

7. ManyOne Networks, http://www.manyone.net/; Digital Universe Foundation, http://www.digitaluniverse.net/.

8. L. Kocarev and G. Vattay (Eds.), Complex Dynamics in Communication Networks (Springer Verlag, Berlin, 2005).

9. J. Horgan, From Complexity to Perplexity, Scientific American, June (1995) 7479.

10. J. Horgan, The End of Science. Facing the Limits of Knowledge in the Twilight of the Scientific Age (Addison-Wesley, Helix, 1996).

11. G.F.R. Ellis, Physics, Complexity and Causality, Nature 435 (2005) 743.

12. G.F.R. Ellis, Physics and the Real World, Physics Today, July (2005) 49-54.

13. A.P. Kirilyuk, Universal Concept of Complexity by the Dynamic Redundance Paradigm: Causal Randomness, Complete Wave Mechanics, and the Ultimate Unifcation of Knowledge (Naukova Dumka, Kyiv, 1997). For a non-technical overview see also Physics/9806002 at http://arXiv.org.

14. A.P. Kirilyuk, Dynamically Multivalued Self-Organisation and Probabilistic Structure Formation Processes, Solid State Phenomena 97-98 (2004) 21-26. Physics/0405063 at http://arXiv.org.

15. A.P. Kirilyuk, Universal Symmetry of Complexity and Its Manifestations at Different Levels of World Dynamics, Proceedings of Institute of Mathematics of NAS of Ukraine 50 (2004) 821-828. Physics/0404006 at http://arXiv.org.

16. A.P. Kirilyuk, The Universal Dynamic Complexity as Extended Dynamic Fractality: Causally Complete Understanding of Living Systems Emergence and Operation, in: Fractals in Biology and Medicine. Vol. III, edited by G.A. Losa, D. Merlini, T.F. Nonnenmacher, and E.R. Weibel (Birkhäuser, Basel, 2002), pp. 271284. Physics/0305119 at http://arXiv.org.

17. A.P. Kirilyuk, Dynamically Multivalued, Not Unitary or Stochastic, Operation of Real Quantum, Classical and Hybrid Micro-Machines, Physics/0211071 at http://arXiv.org.

18. A.P. Kirilyuk, Complex-Dynamical Extension of the Fractal Paradigm and Its Applications in Life Sciences, in: Fractals in Biology and Medicine. Vol. IV, edited by G.A. Losa, D. Merlini, T.F. Nonnenmacher, and E.R. Weibel (Birkhäuser, Basel, 2005), pp. 233-244. Physics/0502133 at http://arXiv.org.

19. A.P. Kirilyuk, Complex Dynamics of Real Nanosystems: Fundamental Paradigm for Nanoscience and Nanotechnology, Nanosystems, Nanomaterials, Nanotechnologies 2 (2004) 1085-1090. Physics/0412097 at http://arXiv.org.

20. A.P. Kirilyuk, Emerging Consciousness as a Result of Complex-Dynamical Interaction Process, Report at the EXYSTENCE workshop Machine Consciousness: Complexity Aspects (Turin, 29 Sep - 1 Oct 2003). Physics/0409140 at http://arXiv.org. 
21. A.P. Kirilyuk, Complex Dynamics of Autonomous Communication Networks and the Intelligent Communication Paradigm, Report at WAC 2004, see Ref. [2]. Physics/0412058 at http://arXiv.org.

22. A.P. Kirilyuk, Unreduced Dynamic Complexity, Causally Complete Ecology, and Realistic Transition to the Superior Level of Life, Report at the conference "Nature, Society and History" (Vienna, 30 Sep - 2 Oct 1999), see http://hal.ccsd.cnrs.fr/ccsd-00004214.

23. A.P. Kirilyuk, Quantum Field Mechanics: Complex-Dynamical Completion of Fundamental Physics and Its Experimental Implications, Physics/0401164 at http://arXiv.org.

24. A.P. Kirilyuk, Complex-Dynamic Cosmology and Emergent World Structure, Report at the International Workshop on Frontiers of Particle Astrophysics (Kiev, 21-24 June 2004). Physics/0408027 at http://arXiv.org.

25. A.P. Kirilyuk, Quantum Chaos and Fundamental Multivaluedness of Dynamical Functions, Annales de la Fondation Louis de Broglie 21 (1996) 455-480. Quantph/9511034-36 at http://arXiv.org.

26. A.P. Kirilyuk, Theory of Charged Particle Scattering in Crystals by the Generalized Optical Potential Method, Nucl. Instr. and Meth. B 69 (1992) 200-231.

27. P.H. Dederichs, Dynamical Diffraction Theory by Optical Potential Methods, in: Solid State Physics: Advances in Research and Applications, Vol. 27, edited by H. Ehrenreich, F. Seitz, and D. Turnbull (Academic Press, New York, 1972), pp. 136-237.

28. A.P. Kirilyuk, 75 Years of the Wavefunction: Complex-Dynamical Extension of the Original Wave Realism and the Universal Schrödinger Equation, Quantph/0101129 at http://arXiv.org. 